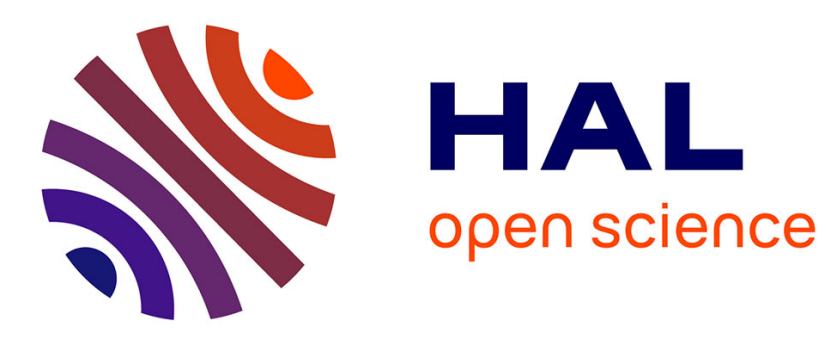

\title{
Backhaul-Constrained Resource Allocation and 3D Placement for UAV-Enabled Networks
}

Marie-Josépha Youssef, Charbel Abdel Nour, Joumana Farah, Catherine

Douillard

\section{- To cite this version:}

Marie-Josépha Youssef, Charbel Abdel Nour, Joumana Farah, Catherine Douillard. BackhaulConstrained Resource Allocation and 3D Placement for UAV-Enabled Networks. VTC2019Fall: IEEE 90th Vehicular Technology Conference, Sep 2019, Honolulu, Hawaii, United States. 10.1109/VTCFall.2019.8891385 . hal-02165343

\section{HAL Id: hal-02165343 \\ https://hal.science/hal-02165343}

Submitted on 10 Oct 2019

HAL is a multi-disciplinary open access archive for the deposit and dissemination of scientific research documents, whether they are published or not. The documents may come from teaching and research institutions in France or abroad, or from public or private research centers.
L'archive ouverte pluridisciplinaire HAL, est destinée au dépôt et à la diffusion de documents scientifiques de niveau recherche, publiés ou non, émanant des établissements d'enseignement et de recherche français ou étrangers, des laboratoires publics ou privés. 


\title{
Backhaul-Constrained Resource Allocation and 3D Placement for UAV-Enabled Networks
}

\author{
Marie-Josepha Youssef ${ }^{(1)}$, Charbel Abdel Nour ${ }^{(1)}$, Joumana Farah ${ }^{(2)}$ and Catherine Douillard ${ }^{(1)}$ \\ (1) IMT Atlantique, Department of Electronics, Lab-STICC - UMR 6285 \\ Technopôle Brest Iroise, CS 83818 - 29238 Brest Cedex, France \\ (2) Department of Electricity and Electronics, Faculty of Engineering, \\ Lebanese University, Roumieh, Lebanon
}

\begin{abstract}
Unmanned aerial vehicle (UAV)-base stations (BS) are envisioned to provide wireless access to areas where the existing wireless infrastructure is either not deployed, damaged or simply congested. In this paper, the problem of minimizing the transmit power of a UAV-BS, while serving users with their QoS requirements and accounting for the wireless backhaul limitation of the UAV, is investigated. To this end, an algorithm that finds the optimal bandwidth assignment in the backhaul link, the optimal 3D position of the UAV, as well as the transmit power distribution in the access and the backhaul links is proposed. Simulation results show that, using the proposed approach, the needed power is greatly reduced, when compared to a strategy that separates between the frequency band used in the access and the backhaul links. In addition, the performance enhancement of a UAV-enabled system over a traditional one in which the MBS directly serves users is shown.
\end{abstract}

Index Terms-UAV-BS, wireless backhaul, in-band full-duplex, QoS requirements.

\section{INTRODUCTION}

The use of unmanned aerial vehicles (UAV) as flying base stations (BS) in wireless communication systems has received a lot of attention in recent literature [1]. Owing to their mobility, flexibility and low cost, UAV-BSs can help increase the throughput and coverage of traditional communication systems [2]. They can also help alleviate traffic congestion in hotspot areas, as well as establish communication links in remote and disaster areas where the existing communication infrastructure is either non-existent or damaged [3].

Accounting for the limited on-board energy of the UAV, the work in [4] investigated the optimal location of the UAV to minimize its transmit power. The authors in [5] evaluated the optimal UAV altitude that maximizes its coverage region. In [6], the authors built on the results of [5] and found the 3D location of the UAV that maximizes the coverage for users having different QoS requirements.

However, none of the works in [4]-[6] accounted for the backhaul link of UAVs. Contrary to most terrestrial BSs, UAVs are connected to the core network through a wireless backhaul link [3]. To be able to reap the benefits promised by the use of UAVs in communication systems, there is a need to dynamically manage this backhaul link according to the traffic state of the network [1]. In fact, if not configured properly, the backhaul link may introduce interference, thus limiting the throughput provided by the UAV in the access link. To increase the spectral efficiency and reduce the latency of systems relying on a wireless backhaul, in-band full-duplex (IBFD) communications were recently investigated [7]. IBFD allows the simultaneous transmission and reception of backhaul and access information in the same frequency band, at the expense of a self-interference (SI), caused by the transmitter to its own receiver. That said, SI cancellation schemes have progressed significantly [8], allowing an efficient application of IBFD for wireless backhauling.

Few works have addressed the wireless backhaul link in the context of UAV-enabled networks. The authors in [3] investigated the 3D placement problem of the UAV for the sake of maximizing the number of served users while considering a backhaul link with constant transmission rate. [9] introduced a heuristic algorithm that finds the number of needed UAVs as well as their 3D positions and accounts for the backhaul constraint. However, the authors did not elaborate on the way bandwidth assignment in the backhaul link is conducted. In [10], the authors proposed an algorithm to find the 3D position of the UAVs as well as the user assignment and bandwidth allocation to maximize the logarithmic rates of users. Nevertheless, they assumed that access and backhaul transmissions take place on different and sufficiently spaced frequency bands to avoid SI.

In this paper, we consider a scenario where the traditional wireless infrastructure is missing, e.g. in remote areas or due to a disaster or BS failure. A UAV is dispatched to serve users having QoS requirements in that area, and an in-band wireless backhaul link is established between the UAV and a macro base station (MBS) to provide the needed backhaul capacity. To minimize the UAV transmit power, an optimization problem that finds the assignment of subbands in the backhaul link, the $3 \mathrm{D}$ position of the UAV as well as the power levels in the access and backhaul links is solved.

The rest of this paper is organized as follows. In sections II and III, the system model and the problem formulation are described. Section IV introduces the proposed solution. Finally, simulation results are presented in Section V, before drawing the conclusions in Section VI.

\section{SySTEM MODEL}

Consider a downlink UAV-enabled communication network consisting of one IBFD-enabled UAV and $K$ users. The UAV is connected to the core network through an MBS $\mathcal{M}$, located at the origin, via wireless in-band backhauling. 
Let $\mathcal{K}$ denote the set of users, and let $R_{k}^{\text {req }}$ and $\left(x_{k}, y_{k}\right)$ indicate the rate requirement and the location of user $k \in$ $\mathcal{K}$, respectively. Also, let the UAV position be denoted by $\boldsymbol{z}_{\boldsymbol{U} \boldsymbol{A} \boldsymbol{V}}=\left(x_{U A V}, y_{U A V}, H\right)$, where $\left(x_{U A V}, y_{U A V}\right)$ refers to the horizontal position of the UAV, while $H$ refers to its altitude. Clearly, the UAV should be positioned to serve as many users as possible, while being able to receive the necessary rate from the backhaul link.

Due to the IBFD wireless backhaul assumption, a user associated with the UAV suffers from the interference of the backhaul link occurring on the same frequency band. Moreover, because of the IBFD nature of the UAV, the latter suffers from SI.

\section{A. Path Loss Model}

For the air-to-ground (A2G) communication between the UAV and its users, two types of path loss are considered: lineof-sight (LOS) and non-line-of-sight (NLOS). The probability of having a LOS communication link between the UAV and user $k$ is given by [11]:

$$
P_{L O S}=\frac{1}{1+\alpha \exp \left(-\beta\left(\frac{180 \theta_{k}}{\pi}-\alpha\right)\right)},
$$

where $\theta_{k}=\arctan \left(\frac{H}{r_{k}}\right)$ is the elevation angle and $r_{k}=$ $\sqrt{\left(x_{k}-x_{U A V}\right)^{2}+\left(y_{k}-y_{U A V}\right)^{2}}$ is the horizontal distance between user $k$ and the UAV. In (1), $\alpha$ and $\beta$ are constants determined by the environment (rural, urban, etc). The NLOS probability is hence: $P_{N L O S}=1-P_{L O S}$.

Since classifying a link as LOS or NLOS is not straightforward and requires terrain knowledge, the probabilistic mean path loss is adopted [6]:

$$
\begin{aligned}
L\left(H, r_{k}\right) & =\eta_{L} \times P_{L O S}+\eta_{N L} \times P_{N L O S}+20 \log \left(\frac{4 \pi f_{c} d_{k}}{c}\right) \\
& =\frac{A}{1+\alpha \exp \left(-\beta\left(\frac{180 \theta_{k}}{\pi}-\alpha\right)\right)}+20 \log \left(\frac{r_{k}}{\cos \left(\theta_{k}\right)}\right)+B .
\end{aligned}
$$

In (2), $20 \log \left(\frac{4 \pi f_{c} d_{k}}{c}\right)$ is the free space path loss, with $f_{c}$ and $c$ being the carrier bandwidth and the speed of light respectively. $\eta_{L}$ and $\eta_{N L}$ are the mean additional losses for LOS and NLOS links, respectively. $d_{k}=\sqrt{\left(r_{k}^{2}+H^{2}\right)}$ is the $3 \mathrm{D}$ distance between the UAV and user $k . A=\eta_{L}-\eta_{N L}$ and $B=20 \log \left(\frac{4 \pi f_{c}}{c}\right)+\eta_{N L}$.

\section{B. Communication Model}

The total frequency bandwidth $B W$ is partitioned into a set $\mathcal{S}$ of $S$ subbands, leading to a subband bandwidth of $B_{c}=$ $B W / S$. It is assumed that $K=S$ and that each user has access to one subband only.

Let $s$ be the subband allocated to user $k$ and let $G_{k, s}$ be the channel gain between the MBS and each user $k$ over subband $s$ consisting of both small-scale and large-scale fading. The rate achieved by user $k$ over $s$ is given by [9]:

$$
R_{k, s}=B_{c} \log _{2}\left(1+\frac{P_{U A V, s} G_{U A V, k}^{2}}{N_{0} B_{c}+a_{s} P_{M B S, s} G_{k, s}^{2}}\right) .
$$

In (3), $P_{U A V, s}$ and $P_{M B S, s}$ are the transmit power on subband $s$ for the UAV and the MBS, respectively, and $N_{0}$ is the noise power spectral density. $G_{U A V, k}^{2}=10^{-L\left(H, r_{k}\right) / 10}$ is the channel gain between the UAV and user $k . a_{s} \in\{0,1\}$ is a binary decision variable such that $a_{s}=1$ if subband $s$ is used in the backhaul link, and 0 otherwise. $P_{M B S, s} G_{k, s}^{2}$ denotes the backhaul interference (BI) suffered by user $k$ when $a_{s}=1$.

The channel between the UAV and the MBS also follows the A2G path loss model. When $a_{s}=1$, the rate achieved by the UAV over $s$ is given by:

$$
R_{U A V, s}=B_{c} \log _{2}\left(1+\frac{P_{M B S, s} G_{U A V, M B S}^{2}}{N_{0} B_{c}+C_{S I} P_{U A V, s}}\right) .
$$

In (4), $G_{U A V, M B S}^{2}$ is the channel gain between the UAV and the MBS and $C_{S I} P_{U A V, s}$ is the residual SI experienced at the UAV on subband $s$.

\section{PROBLEM FORMULATION}

Since UAVs are energy-limited, the objective of the optimization problem is to minimize the transmit power of the UAV while meeting the rate requirements of all users:

$$
\begin{aligned}
& \min _{\boldsymbol{P}_{\boldsymbol{U} \boldsymbol{A} \boldsymbol{V}}, \boldsymbol{P}_{M \boldsymbol{S}}, \boldsymbol{z}_{\boldsymbol{U} \boldsymbol{A} \boldsymbol{V}}, \boldsymbol{a}, \boldsymbol{b}} \sum_{k \in \mathcal{K}} \sum_{s \in \mathcal{S}} b_{k, s} P_{U A V, s} \\
& \text { such that } \\
& \sum_{s \in \mathcal{S}} b_{k, s} R_{k, s} \geq R_{k}^{\mathrm{req}}, \forall k \in \mathcal{K}, \\
& \sum_{k \in \mathcal{K}} \sum_{s \in \mathcal{S}} b_{k, s} R_{k, s} \leq \sum_{s \in \mathcal{S}} a_{s} R_{U A V, s}, \\
& \sum_{s \in \mathcal{S}} P_{U A V, s} \leq P_{U A V}^{\max }, \\
& \sum_{s \in \mathcal{S}} a_{s} P_{M B S, s} \leq P_{M B S}^{\max }, \\
& H_{\min } \leq H \leq H_{\max }, \\
& a_{s}, b_{k, s} \in\{0,1\} .
\end{aligned}
$$

In (5), $b_{k, s}$ is a binary variable that is equal to 1 if user $k$ is scheduled on subband $s$ and 0 otherwise. (5a) is the rate requirement constraint for each user, while (5b) is the backhaul data rate constraint which ensures that the total rate delivered by the UAV to its assigned users does not exceed its backhaul capacity. In (5c) and (5d), $P_{U A V}^{\max }$ and $P_{M B S}^{\max }$ are the power budgets of the UAV and the MBS respectively. (5e) is the altitude constraint for the UAV.

Problem (5) consists of three subproblems: the UAV placement problem, as well as the bandwidth and power allocation in the access and backhaul links. One can see that, at the optimum, constraint (5a) is satisfied with equality for all users. Hence, the left hand side of (5b) can be substituted with $\sum_{k} R_{k}^{\text {req }}$. Nonetheless, the resulting formulated optimization problem is mixed-integer and multivariate. To solve it, we propose a multi-step algorithm that targets the three subproblems.

If the assignment of subbands in the access link, $\boldsymbol{b}$, is known, solving (5) resorts to finding the assignment of backhaul subbands $\boldsymbol{a}$, the UAV position $\boldsymbol{z}_{\boldsymbol{U} \boldsymbol{A} \boldsymbol{V}}$ and the power variables $\boldsymbol{P}_{U A V}$ and $\boldsymbol{P}_{M B S}$ that minimize the UAV transmit power. Therefore, the proposed solution proceeds as follows: 
1) Find the 2D UAV deployment region that meets rate constraints of the users (5a) and (5b).

2) Decide on the assignment of backhaul subbands. To do so:

a) Make an initial assumption on the minimum number of required backhaul subbands. This initial assumption does not take into account the impact of the resulting interference at the user side.

b) At a second stage, a set of admissible subbands is created. Admissible subbands are the ones ensuring constraints (5a) through (5d). Their selection takes into account the resulting interference at the user side.

c) Finally, from the set of admissible subbands, the solution minimizing the interference at the user side is retained.

3) Solve the exact positioning problem for the UAV.

4) Optimize the power variables in the access and backhaul links.

Steps 1 and 2 of the solution are mutually dependent. In fact, depending on the choice of backhaul subbands, users experience a different level of BI which affects the acceptable region in which the UAV can be located in order to ensure their requirements. After settling on the potential UAV deployment region and the assignment of backhaul subbands, the exact position of the UAV and the power variables are found.

Note that in this paper, we assume that the available UAV power is sufficient for guaranteeing the QoS requirements of all $K$ users and leave the case where it is not for future work.

\section{PRoposed Solution}

As stated in Section III, finding the acceptable placement region of the UAV and deciding on the assignment of backhaul subbands are mutually dependent. Moreover, deciding on the set of backhaul subbands depends on the power level per subband in the access and the backhaul link. Therefore, as a necessary intermediate step, we assume an equal power repartition in the access and backhaul links:

$$
\begin{gathered}
P_{U A V, s}=P_{U A V}^{\max } / S, \forall s \in \mathcal{S}, \\
P_{M B S, s}=P_{M B S}^{\text {needed }} / \sum_{s \in \mathcal{S}} a_{s}, \forall s \in \mathcal{S} .
\end{gathered}
$$

In (7), $P_{M B S}^{\text {needed }}$ is the needed MBS power to satisfy the backhaul capacity requirement; it should satisfy $P_{M B S}^{\text {needed }} \leq P_{M B S}^{\max }$. The derivation of $P_{M B S}^{\text {needed }}$ will be further discussed in Section IV-B. Note that this equal power repartition might result in some users exceeding their rate requirements. Therefore, at the end of the proposed algorithm, a power optimization step will be conducted to ensure that the rate requirements of users are met with equality, thus replacing the equal power assumption.

Next, the first step of the proposed solution that aims at finding the 2D UAV deployment region guaranteeing constraints (5a) and (5b) is described.

\section{A. Finding the coverage region for each user and for the MBS}

If power and subband allocation in the access link are known, the rate requirement in (5a) can be translated into a requirement on the maximum tolerable path loss as follows:

$$
L\left(H, r_{k}\right) \leq-10 \log _{10}\left\{\left(2^{\frac{R_{k}^{\text {req }}}{B_{c}}}-1\right) \frac{N_{0} B_{c}+a_{s} P_{M B S, s} G_{k, s}^{2}}{P_{U A V, s}}\right\} .
$$

In other words, user $k$ meets its rate requirement if its experienced path loss $L\left(H, r_{k}\right)$ is less than or equal to the maximum tolerable path loss $L_{k}^{\text {th }}$, where $L_{k}^{\text {th }}$ is the right hand side of (8). For a given UAV altitude $H$, let $C_{k}(H)$ be the maximum 2D distance between the UAV and user $k$ guaranteeing $R_{k}^{\text {req }}$, i.e. $C_{k}(H)=\left.r\right|_{L_{(H, r)}=L_{k}^{\text {th }}}$.

From (2), for a given UAV altitude $H$, if the UAV is located at a distance $C_{k}(H)$ from user $k$, the latter experiences a constant path loss $L_{k}^{\text {th }}$. Moreover, if the UAV is located at a distance $r \leq C_{k}(H)$ from user $k$, the latter experiences a path loss $L(H, r) \leq L_{k}^{\text {th }}$. Hence, from the perspective of user $k$, its rate requirement is met if the UAV is positioned inside the circular disk having user $k$ as center and $C_{k}(H)$ as radius. It was shown in [5] that the coverage radius of the UAV as a function of $H$ has one optimum point only which corresponds to the largest coverage radius. In this work, we propose to make use of the inverse relation which equally holds, since the channel between the UAV and the users is assumed symmetrical. In other words, from the perspective of user $k$, the coverage radius $C_{k}(H)$ as a function of $H$ has one optimum point corresponding to the largest coverage radius. Hence, finding $C_{k}(H)$ consists of finding the optimal altitude that results in the maximum coverage region. It was also shown in [5], [6] that finding the optimal altitude consists of solving $\frac{\partial \theta}{\partial H}=0$ and that $\theta_{\text {opt }}$ that maximizes $C_{k}(H)$ depends only on the considered environment, leading to: $\theta_{\text {opt }}=20.34^{\circ}, 42.44^{\circ}, 54.62^{\circ}$ for the suburban, urban and dense-urban environments respectively.

Having the optimal elevation angle $\theta_{\text {opt }}$, the largest coverage radius of user $k, C_{k}$ can be found by solving:

$$
L_{k}^{\mathrm{th}}=\frac{A}{1+\alpha \exp \left(-\beta\left(\frac{180 \theta_{\mathrm{opt}}}{\pi}-\alpha\right)\right)}+20 \log \left(\frac{C_{k}}{\cos \left(\theta_{\mathrm{opt}}\right)}\right)+B .
$$

Hence, we propose to associate every user $k$ with a 2D coverage region $C_{k}$ that guarantees its rate requirement. Let $D_{k}$ be the disc centered at user $k$ and having $C_{k}$ as radius. It should be noted that $C_{k}$ will only be used to find the acceptable $2 \mathrm{D}$ region that can successfully serve all users simultaneously.

Similarly, when considering the backhaul link, the UAV should be positioned in a region where it is able to receive the required backhaul rate from the MBS. Recall that equal power repartition is assumed in the access and the backhaul links. Therefore, $R_{U A V, s}$, the rate achieved by the UAV on subband $s$ in the backhaul link, is the same for all subbands used in the backhaul link, since it depends only on the path loss between the UAV and the MBS. Let $S_{B H}$ be the value of the total number of backhaul subbands, $R_{U A V, s}$ is computed 
as:

$$
R_{U A V, s}=\sum_{k \in \mathcal{K}} R_{k}^{\mathrm{req}} / S_{B H}
$$

To meet the required backhaul rate, the path loss between the UAV and the MBS should satisfy the following condition:

$$
\begin{aligned}
L\left(H, r_{M B S}\right) \leq & -10 \log _{10}\left\{\left(2^{\frac{R_{U A V, s}}{B_{c}}}-1\right) \times\right. \\
& \left.\frac{N_{0} B_{c}+C_{S I} P_{U A V, s}}{P_{M B S, s}}\right\}=L_{S_{B H}}^{\mathrm{th}} .
\end{aligned}
$$

As in the users case, the maximum coverage region for the MBS satisfying the backhaul path loss requirement is found using (9) after replacing $L_{k}^{\text {th }}$ with $L_{S_{B H}}^{\text {th }}$. Since the coverage radius of the MBS is a function of the number of backhaul subbands, it is denoted by $C_{M B S, S_{B H}}$. Let $D_{M B S, S_{B H}}$ be the disc centered at the origin and having $C_{M B S, S_{B H}}$ as radius.

Having found the $2 \mathrm{D}$ region guaranteeing (5a) and (5b), the set of backhaul subbands numbers ensuring the intersection of the different coverage regions is found next. Also, for each value of backhaul subbands $S_{B H}$, a metric that decides on the specific choice of these subbands is devised.

\section{B. Finding the set of admissible backhaul subbands}

To ensure that the UAV can provide the access rate to its users while meeting its backhaul rate requirement, it must be positioned in the intersection of the coverage region of all users and that of the MBS. Nonetheless, the coverage region of the MBS depends on the number of subbands used in the backhaul link, i.e. on $S_{B H}$. When the latter is small, the required data rate per subband, $R_{U A V, s}$, is large. This causes the maximum tolerable path loss $L_{S_{B H}}^{\text {th }}$ to be small, resulting in a narrow MBS coverage radius. On the other hand, if all subbands are used in the backhaul link, the MBS coverage radius becomes large. However, for some users with high rate requirements, the added BI shrinks their coverage regions. This could result in an impossibility to serve all users simultaneously if the added interference proves excessive for some. Therefore, the number and the choice of backhaul subbands should be optimized.

To find the number of backhaul subbands that results in the largest intersection of coverage regions, for each potential value of the number of backhaul subbands $S_{B H}$, i.e. for $S_{B H}=1, \ldots, S$, the corresponding backhaul subbands must be chosen, and the simultaneous intersection region must be found. However, to reduce the values of backhaul subbands numbers to be tested, the following observation is made.

Proposition 1. The largest simultaneous coverage region for the users, $D_{\text {int }}^{\max }$, is achieved when none of the subbands is used in the backhaul link, i.e. when $a_{s}=0, \forall s \in \mathcal{S}$.

Proof. Let $D_{\text {int }}$ be the simultaneous coverage region when a subband $s$ is used in the backhaul link and let $k$ be the user scheduled on $s$. According to (8), $L_{k}^{\text {th }}$ decreases when the BI increases. Hence, the coverage region of $k$ becomes smaller when its allocated subband is used in the backhaul link, in comparison to the opposite case. Therefore, the simultaneous coverage region $D_{\text {int }}$ is smaller than $D_{\text {int }}^{\max }$, when one or more subbands are used in the backhaul link.

Following Proposition 1, any MBS coverage region that does not intersect with $D_{\text {int }}^{\max }$ does not intersect with the simultaneous user coverage region that accounts for BI. Let $\left(x_{0}, y_{0}\right) \in D_{\text {int }}^{\max }$ be the closest point in 2D space to the MBS, i.e. $\left(x_{0}, y_{0}\right)=\underset{(x, y) \in D_{\text {int }}^{\max }}{\operatorname{argmin}} \sqrt{x^{2}+y^{2}}$. In order to have an intersection with $D_{\text {int }}^{\max }$, the MBS coverage radius must satisfy:

$$
C_{M B S, S_{B H}} \geq C_{M B S, \min }=\sqrt{x_{0}^{2}+y_{0}^{2}} .
$$

The minimum value of the number of subbands to be used in the backhaul link, $S_{\min , B H}$, is the one satisfying (12) and is found using bisection search. For every value $S_{B H} \geq S_{\min , \mathrm{BH}}$, the coverage radius satisfies $C_{M B S, S_{B H}} \geq C_{M B S \text {, min }}$.

When $S_{B H}$ is small, so is $L_{S_{B H}}^{\mathrm{th}}$, and hence, the MBS must use all its power budget in order to achieve the required path loss, i.e. $P_{M B S, s}=\frac{P_{M B S}^{\max }}{S_{B H}}$. With the increase of $S_{B H}$ and $L_{S_{B H}}^{\mathrm{th}}, C_{M B S, S_{B H}}$ increases to the point where the backhaul coverage region encompasses the coverage regions of all users. However, the UAV location should not be very far from the MBS since it would logically require more backhaul power to reach its backhaul rate requirement. This translates into more $\mathrm{BI}$ at the user side, which in its turn increases the needed UAV power to guarantee the access rate requirements. Therefore, the following observation is made.

Proposition 2. The maximum needed backhaul coverage radius, $C_{M B S, \max }$, is given by the distance between the MBS and the user farthest from it.

When using the whole MBS power budget, if $S_{B H}$ results in a backhaul coverage radius larger than $C_{M B S \text {,max }}$, the needed backhaul power is reduced in order to ensure a coverage radius of $C_{M B S \text {,max }}$. To find the needed backhaul power for $S_{B H}$ backhaul subbands, the maximum path loss, $L_{M B S, \max }$, is first found using (9) after setting $C_{k}=C_{M B S \text {,max }}$. The power per backhaul subband is then given by:

$$
P_{M B S, s}=\left(2^{\frac{R_{U A V, s}}{B c}}-1\right) \frac{N_{0} B_{c}+C_{S I} P_{U A V, s}}{10^{-L_{M B S, \max } / 10}} .
$$

Hence, $P_{M B S}^{\text {needed }}$ is given by: $P_{M B S}^{\text {needed }}=P_{M B S, s} \times S_{B H}$. When $S_{B H}$ increases while maintaining the MBS coverage radius at $C_{M B S \text {,max }}$, the backhaul power needed per subband decreases. Therefore, $P_{M B S}^{\text {needed }}<P_{M B S}^{\max }$.

Having found the minimum required number of backhaul subbands and the backhaul power needed for every potential value of backhaul subbands number, we now turn our attention to the choice of these subbands. Clearly, this choice should be done carefully since it has an undeniable impact on the interference levels experienced by the users. To this end, a metric is introduced for the choice of the backhaul subbands.

As shown in (8), every user has an upper bound on its acceptable path loss $L_{k}^{\text {th }}$. The latter depends on the requested data rate of user $k$, the BI and the power of the UAV on the subband assigned to $k$. To tend towards the best achievable performance (i.e. lowest interference levels), the upper bounds 
of all users should be as high as possible, since a larger upper bound reflects in a larger coverage region.

Therefore, the backhaul subbands should be chosen to guarantee that the resulting upper bounds are as large as possible. For user $k, L_{k}^{\text {th }}$ increases when the term inside the brackets in (8) decreases. Therefore, each user $k$ is associated with the following metric:

$$
M(k)=\left(2^{\frac{R_{k}^{\mathrm{req}}}{B_{c}}}-1\right)\left(N_{0} B_{c}+P_{M B S, s} G_{k, s_{k}}^{2}\right) .
$$

Note that $P_{U A V, s}$ is removed from (14) since it is common to all users.

To optimize system performance when $S_{B H}$ subbands are used in the backhaul link, the subbands belonging to the $S_{B H}$ users having the lowest metric given by (14) are chosen.

Once the backhaul subbands are known, the path loss upper bounds taking into account the BI at the user side can be calculated for all users according to (8), for all $S_{B H} \geq S_{\min , \mathrm{BH}}$. Let the set of path loss upper bounds be denoted by $L^{\mathrm{ub}} \in \mathbb{R}^{K \times\left|\mathcal{S}_{B H}^{\text {poss }}\right|}$, where $\mathcal{S}_{B H}^{\text {poss }}$ is the set containing the potential values of backhaul subbands numbers, i.e. $\mathcal{S}_{B H}^{\text {poss }}=\left\{S_{B H, \min }, S_{B H, \text { min }}+1, \ldots, S\right\}$.

In the next subsection, we will solve for the value of $S_{B H}$ that ensures the best system performance.

\section{Deciding on the final number of backhaul subbands}

The minimum number of backhaul subbands $S_{B H \text {,min }}$ was found without considering BI. When accounting for this interference, the coverage regions of users decrease in size. Therefore, it is not guaranteed that all subbands numbers in $\mathcal{S}_{B H}^{\text {poss }}$ ensure that the coverage regions of users intersect with that of the MBS on one hand, and with each other on the other. Hence, in this subsection, an algorithm to find the value of the number of backhaul subbands resulting in the best performance is introduced.

First, to guarantee the intersection of the MBS coverage region with that of all users, the following observation is made.

Proposition 3. For $S_{B H}$ backhaul subbands, the coverage region of the MBS intersects with that of user $k$ if (15) holds:

$$
L_{k, S_{B H}}^{\mathrm{ub}} \geq L_{k, S_{B H}}^{\min } .
$$

Proof. The coverage region of the MBS intersects with that of user $k$ if (16) holds:

$$
C_{k, S_{B H}} \geq C_{k, S_{B H}}^{\min }=\sqrt{x_{k}^{2}+y_{k}^{2}}-C_{M B S, S_{B H}} .
$$

From $C_{k, S_{B H}}^{\min }$, we can find the minimum accepted path loss $L_{k, S_{B H}}^{\min }$ which results in the intersection of the two coverage regions. $L_{k, S_{B H}}^{\min }$ relates to the maximum accepted rate requirement for user $k$ in order for the UAV to be able to serve him, when $S_{B H}$ subbands are used in the backhaul link. Having $L^{\mathrm{ub}}$ as the upper bound on achieved path loss for all potential values $S_{B H} \in \mathcal{S}_{B H}^{\text {poss }}$, the coverage region of user $k$ intersects with that of the MBS if (15) holds.
Hence, any potential value of $S_{B H}$ that does not verify condition (15) for all users is removed from $\mathcal{S}_{B H}^{\text {poss }}$.

Although all values of $S_{B H}$ remaining in $\mathcal{S}_{B H}^{\text {poss }}$ guarantee that the coverage region of each user intersects with that of the MBS, not all of them guarantee that the coverage regions of users intersect with each other. In other words, not all of these potential values guarantee that the users can be served simultaneously while receiving the necessary information from the MBS. Therefore, an algorithm that finds the number of backhaul subbands resulting in the best coverage of all users simultaneously is introduced.

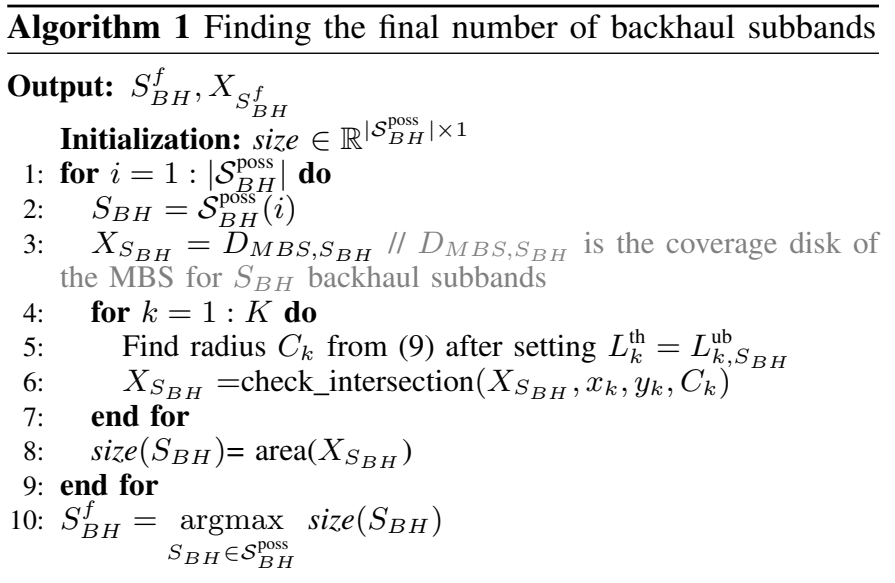

Algorithm 1 finds, for each $S_{B H} \in \mathcal{S}_{B H}^{\text {poss }}$, the area of the intersection region of all users (Lines 4 through 8 ). The number of backhaul subbands guaranteeing service to all users simultaneously are those having size $>0$. After finding the intersection area for every $S_{B H} \in \mathcal{S}_{B H}^{\text {poss }}$, the number that maximizes the simultaneous coverage region, $S_{B H}^{f}$, as well as this coverage region $X_{S_{B H}^{f}}$, are retained for the subsequent steps.

\section{Finding the optimal 3D UAV position and the power levels}

To minimize the UAV transmit power, problem (5) is reformulated as follows:

$$
\begin{aligned}
& \min _{\boldsymbol{P}_{U A \boldsymbol{V}}, \boldsymbol{P}_{M B S}, \boldsymbol{z}_{U A V}} \sum_{k \in \mathcal{K}} \sum_{s \in \mathcal{S}} b_{k, s} P_{U A V, s} \\
& \text { such that } \sum_{s \in \mathcal{S}} b_{k, s} R_{k, s}=R_{k}^{\text {req }}, \forall k \in \mathcal{K} \text {, } \\
& \sum_{k \in \mathcal{K}} \sum_{s \in \mathcal{S}} b_{k, s} R_{k, s} \leq \sum_{s \in \mathcal{S}} a_{s} R_{U A V, s}, \\
& \sum_{s \in \mathcal{S}} a_{s} P_{M B S, s} \leq P_{M B S}^{\max }, \\
& H_{\text {min }} \leq H \leq H_{\max } \text {, } \\
& \left(x_{U A V}, y_{U A V}\right) \in X_{S_{B H}^{f}} \text {. }
\end{aligned}
$$

(17e) states that the 2D position of the UAV should be in the simultaneous coverage region of the users and the MBS.

Increasing the BI causes an increase in the access power in order to maintain the needed access rate requirements. Therefore, to minimize the needed access power, the BI, hence the backhaul power, should be kept as low as possible, while 
at the same time meeting the backhaul rate requirement. To this end, the constraint in (17b) should be met with equality.

Let $s_{k}$ be the subband assigned to user $k$. From (3), the value of $P_{U A V, s_{k}}$ that satisfies (17a) for each user $k$ is:

$$
\begin{aligned}
P_{U A V, s_{k}} & =\left(2^{\frac{R_{k}^{\mathrm{req}}}{B_{c}}}-1\right)\left(N_{0} B_{c}+a_{s_{k}} P_{M B S, s_{k}} G_{k, s_{k}}^{2}\right) / G_{U A V, k}^{2} \\
& =A_{1}(k)\left(N_{0} B_{c}+a_{s_{k}} P_{M B S, s_{k}} G_{k, s_{k}}^{2}\right) / G_{U A V, k}^{2},
\end{aligned}
$$

where $A_{1}(k)=\left(2^{R_{k}^{\text {req }} / B_{c}}-1\right)$. Note that in (18), the actual value of the elevation angle is used, i.e. $\theta_{k}=\arctan \left(H / r_{k}\right)$.

In (18), $P_{M B S, s_{k}}$ is the backhaul power used by the MBS in order to meet the backhaul rate requirement on subband $s_{k}$. It is given by:

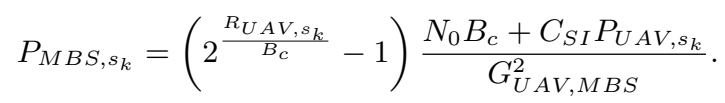

After replacing (19) into (18), $P_{U A V, s_{k}}$ can be expressed as:

$$
P_{U A V, s_{k}}=\frac{A_{1}(k) N_{0} B_{c} \times\left\{G_{U A V, M B S}^{2}+a_{s_{k}} A_{2}(k) G_{k, s_{k}}^{2}\right\}}{G_{U A V, M B S}^{2} G_{U A V, k}^{2}-a_{s_{k}} G_{k, s_{k}}^{2} A_{1}(k) A_{2}(k) C_{S I}} .
$$

In (20), $A_{2}(k)=\left(2^{R_{U A V, s_{k}} / B_{c}}-1\right)$.

Although $\boldsymbol{A}_{\mathbf{1}}$ is known for all users, $\boldsymbol{A}_{\mathbf{2}}$ is not as it depends on the backhaul rate per subband which, in its turn, depends on the power in the backhaul and access links. Therefore, solving (17) is equivalent to solving the following problem.

$$
\begin{aligned}
& \min _{z_{U A \boldsymbol{V}}, \boldsymbol{A}_{\mathbf{2}}} J\left(\boldsymbol{z}_{U \boldsymbol{A V}}, \boldsymbol{A}_{\mathbf{2}}\right)=\sum_{k \in \mathcal{K}} P_{U A V, s_{k}} \\
& \text { such that } \sum_{s \in \mathcal{S}} a_{s} P_{M B S, s} \leq P_{M B S}^{\max }, \\
& H_{\min } \leq H \leq H_{\max }, \\
&\left(x_{U A V}, y_{U A V}\right) \in X_{S_{B H}^{f}},
\end{aligned}
$$

where $P_{U A V, s_{k}}$ is given by (20).

The solution of (21) is divided into two phases. In the first phase, assuming that $\boldsymbol{A}_{\mathbf{2}}$ is known, (21) is solved numerically for the UAV position. Then, with the computed UAV position, the values of $\boldsymbol{A}_{\mathbf{2}}$ that minimize the UAV transmit power are found. The two steps are iterated until convergence. The UAV placement and power optimization technique is summarized in Algorithm 2. Upon convergence of Algorithm 2, the power values in the backhaul and access links are computed according to (19) and (20), respectively.

\footnotetext{
Algorithm 2 Finding the 3D position of the UAV and the power values

Output: $z_{U A V}, P_{U A V}, P_{M B S}$

Initialization: $R_{U A V, s}=\sum_{k \in \mathcal{K}} R_{k}^{\mathrm{req}} / S_{B H}^{f}, \forall s \in \mathcal{S}$ s.t. $a_{s}=1$.

Using $R_{U A V, s} \forall s \in \mathcal{S}$, calculate the initial value of $\boldsymbol{A}_{\mathbf{2}}$.

Repeat:

1: Solve problem (21) for $\boldsymbol{z}_{\boldsymbol{U} A \boldsymbol{V}}$.

2: Using $\boldsymbol{z}_{U \boldsymbol{A} \boldsymbol{V}}$ found in step 1, solve problem (21) for $\boldsymbol{A}_{\mathbf{2}}$.

Until Convergence.

3: Calculate $\boldsymbol{P}_{M B S}$ and $\boldsymbol{P}_{\boldsymbol{U}} \boldsymbol{A V}$ using (19) and (20), respectively.
}

\section{Simulation Results}

In our simulations, users are located within a squared urban area of size $1 \mathrm{~km} \times 1 \mathrm{~km}$, with the MBS located at the bottom left corner. The simulation parameters are listed in Table I, where the urban environment constants are set according to [11].

TABLE I: Simulation Parameters

\begin{tabular}{|c|c|c|c|}
\hline Parameter & Value Range & Parameter & Value Range \\
\hline$(\alpha, \beta)$ & $(9.61,0.16)$ & $\left(\eta_{L}, \eta_{N L}\right)$ & $(1,20) \mathrm{dB}$ \\
\hline$f_{c}$ & $2 \mathrm{GHz}$ & $B W$ & $20 \mathrm{MHz}$ \\
\hline$\left(P_{M B S}^{\max }, P_{U A V}^{\max }\right)$ & $(4,1) \mathrm{W}$ & $\left(H_{\min }, H_{\max }\right)$ & $(100,800) \mathrm{m}$ \\
\hline$K$ & $8,16,32,64$ & $R^{\text {req }}=\sum_{k} R_{k}^{\text {req }}$ & 100 to $180 \mathrm{Mbps}$ \\
\hline $\begin{array}{c}\text { User-MBS } \\
\text { Path Loss }\end{array}$ & $\begin{array}{c}128.1+37.6 \times \\
\log _{10}(d[\mathrm{~km}])\end{array}$ & $\begin{array}{c}\text { RMS delay } \\
\text { spread }\end{array}$ & $500 \mathrm{~ns}$ \\
\hline$C_{S I}$ & $130 \mathrm{~dB}$ & $N_{0}$ & $-174 \mathrm{dBm} / \mathrm{Hz}$ \\
\hline
\end{tabular}

Note that we assume users belong to 4 different QoS classes, each characterized by a distinct rate requirement.

We compare the performance of our proposed method with the traditional case in which the MBS serves users directly through the access link. An algorithm based on the study in [10], denoted by OBA-PSO, is also evaluated. In OBAPSO, the available frequency band is divided orthogonally between the access and backhaul links and the 3D placement of the UAV is conducted using the particle swarm optimization (PSO) algorithm.

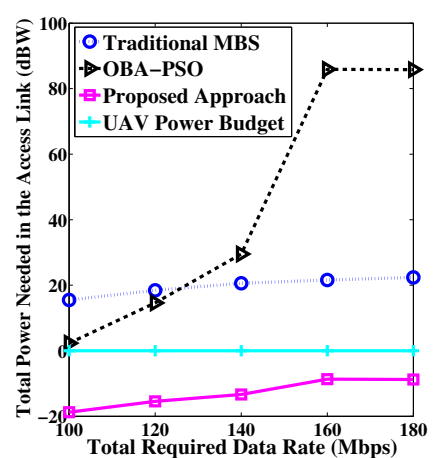

(a) Access Link

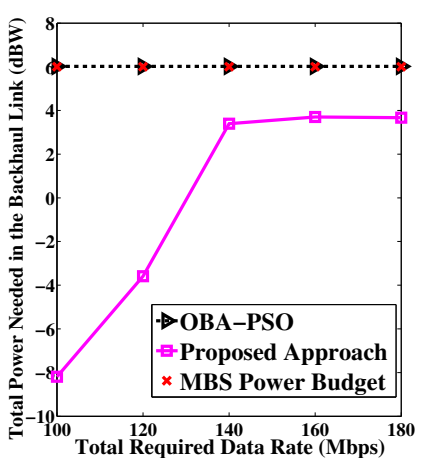

(b) Backhaul Link
Fig. 1: Total power needed as a function of $R^{\text {req }}$ for $K=32$ users.

Fig. 1 shows the total power needed, in $\mathrm{dBW}$, in the access link (Fig. 1a) and the backhaul link (Fig. 1b) in terms of $R^{\text {req }}$ for $K=32$ users. Clearly, having the MBS serve the users directly is not energy-efficient as it needs up to 22.4 $\mathrm{dBW}$ or $173 \mathrm{~W}$ to serve the users when $R^{\text {req }}=180 \mathrm{Mbps}$. For OBA-PSO, even for the lowest rate requirement, the UAV needs $2.3 \mathrm{dBW}$ or $1.7 \mathrm{~W}$ on average to serve the users, which is higher than its power budget. Clearly, OBA-PSO cannot accommodate higher data rates because of the large increase in the needed power in the access link. In fact, when $R^{\text {req }}$ increases, the bandwidth used for the backhaul link does also which decreases the bandwidth available to the access link. Hence, to serve its users, the UAV must increase its power. In contrast, the proposed method can meet all simulated rate requirements while respecting the power budget of the UAV. In fact, in our proposed method, the UAV consumes less than $14 \%$ of its power budget. In the backhaul link, OBA-PSO always uses all the MBS power budget in order to minimize 
the bandwidth needed for the backhaul link. This is not the case in our proposed method which adapts the needed MBS power depending on the needed backhaul capacity.

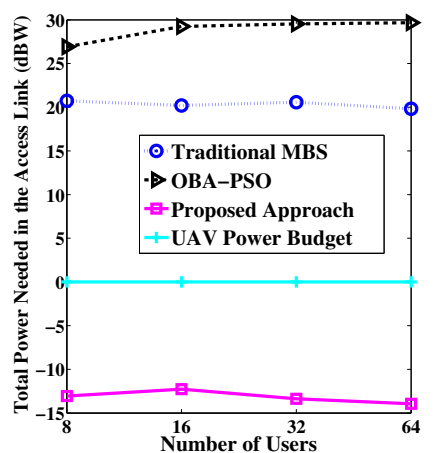

(a) Access Link

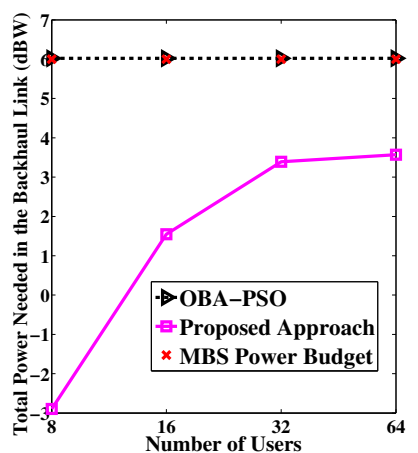

(b) Backhaul Link
Fig. 2: Total power needed as a function of $K$ for $R^{\text {req }}=140$ Mbps.

Fig. 2a shows the needed power in the access link to meet $R^{\text {req }}$ for a varying number of users. While the traditional MBS approach and the OBA-PSO method require a large amount of power (largely exceeding the power budget of the UAV), our proposed method can meet $R^{\text {req }}$ for all $K$ values while using less than $6 \%$ of its power budget. From Fig. $2 b$, we notice that, for our proposed method, the needed power in the backhaul link increases with the number of users, although remaining below the MBS power budget. In fact, when $K$ increases, the bandwidth available to each user becomes smaller. Therefore, to avoid increasing the $\mathrm{BI}$ at the user side, the MBS decreases the power per backhaul subband. However, to meet the needed backhaul capacity, the MBS has to use a larger amount of bandwidth, which therefore increases its consumed power.

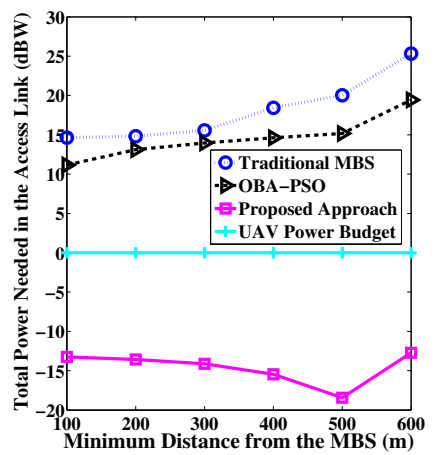

(a) Access Link

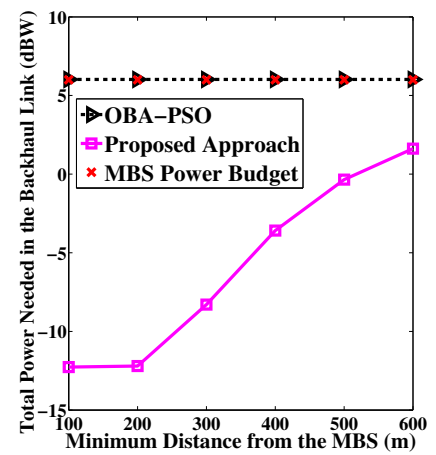

(b) Backhaul Link
Fig. 3: Total power needed as a function of the minimum user-MBS distance for $K=32$ users and $R^{\text {req }}=120 \mathrm{Mbps}$.

Finally, Fig. 3 shows the needed power in terms of the minimum distance between users and the MBS for $K=32$ users. As the minimum user-MBS distance increases, the MBS and the OBA-PSO method need more power to reach $R^{\text {req }}$. In contrast, our proposed method can meet $R^{\text {req }}$ for all minimum distance values, while respecting the UAV power budget. An interesting behavior is shown in Fig. 3a, where the access power decreases to reach a minimum for a minimal distance of $500 \mathrm{~m}$, and increases afterwards. Although the backhaul power (shown in Fig. 3b) increases starting from $200 \mathrm{~m}$, the average channel quality between the MBS and the users is weak when the distances are large, decreasing the experienced BI and hence the needed access power. However, at higher values of the minimum distance such as $600 \mathrm{~m}$, the UAV, needing to remain close enough to the MBS to reach the backhaul capacity, cannot be very close to the users, which increases the power needed in the access link.

\section{CONCLUSION}

In this paper, we introduced a new technique for resource allocation and 3D placement optimization in UAV-enabled networks with backhaul consideration. The proposed technique aims at minimizing the UAV transmit power necessary to meet the rate requirements of the users, while respecting the backhaul capacity and the power budgets of the UAV and the MBS. The results have shown that by adopting the proposed approach, the needed power significantly decreases, compared to the case of a UAV-enabled network that separates between the frequency used in the access and the backhaul link, as well as a traditional network.

\section{ACKNOWLEDGMENT}

This work has been funded with support from IMT Atlantique and the Lebanese University.

\section{REFERENCES}

[1] M. Mozaffari, W. Saad, M. Bennis, Y. Nam, and M. Debbah, "A tutorial on UAVs for wireless networks: Applications, challenges, and open problems," IEEE Commun. Surveys Tuts., pp. 1-28, Mar. 2019.

[2] Y. Zeng, R. Zhang, and T. J. Lim, "Wireless communications with unmanned aerial vehicles: opportunities and challenges," IEEE Commun. Mag., vol. 54, no. 5, pp. 36-42, May 2016.

[3] E. Kalantari, M. Z. Shakir, H. Yanikomeroglu, and A. Yongacoglu, "Backhaul-aware robust 3D drone placement in 5G+ wireless networks," in Proc. Int. Conf. on Communications (ICC) Workshops, Paris, France, May 2017, pp. 109-114.

[4] L. Wang, B. Hu, and S. Chen, "Energy efficient placement of a drone base station for minimum required transmit power," IEEE Wireless Commun. Lett., Early Access, Feb. 2018.

[5] A. Al-Hourani, S. Kandeepan, and S. Lardner, "Optimal LAP altitude for maximum coverage," IEEE Wireless Commun. Lett., vol. 3, no. 6, pp. 569-572, Dec. 2014.

[6] M. Alzenad, A. El-Keyi, and H. Yanikomeroglu, "3-D placement of an unmanned aerial vehicle base station for maximum coverage of users with different QoS requirements," IEEE Wireless Commun. Lett., vol. 7 , no. 1, pp. 38-41, Feb. 2018.

[7] M. Heino, D. Korpi, T. Huusari, E. Antonio-Rodriguez, S. Venkatasubramanian, T. Riihonen, L. Anttila, C. Icheln, K. Haneda, R. Wichman, and M. Valkama, "Recent advances in antenna design and interference cancellation algorithms for in-band full duplex relays," IEEE Commun. Mag., vol. 53, no. 5, pp. 91-101, May 2015.

[8] Y. Choi and H. Shirani-Mehr, "Simultaneous transmission and reception: Algorithm, design and system level performance," IEEE Trans. Wireless Commun., vol. 12, no. 12, pp. 5992-6010, Dec. 2013.

[9] L. Zhang and N. Ansari, "On the number and 3-D placement of inband full-duplex enabled drone-mounted base-stations," IEEE Wireless Commun. Lett., vol. 8, no. 1, pp. 221-224, Feb. 2019.

[10] E. Kalantari, I. Bor-Yaliniz, A. Yongacoglu, and H. Yanikomeroglu, "User association and bandwidth allocation for terrestrial and aerial base stations with backhaul considerations," in Proc. IEEE Annual International Symp. on Personal, Indoor, and Mobile Radio Commun. (PIMRC), Montreal, QC, Canada, Oct. 2017, pp. 1-6.

[11] A. Al-Hourani, S. Kandeepan, and A. Jamalipour, "Modeling air-toground path loss for low altitude platforms in urban environments," in Proc. IEEE Global Commun. Conf. (GLOBECOM), Austin, Tx, USA, Dec. 2014, pp. 2898-2904. 